\title{
Reports and Surveys
}

\section{BRITISH ASSOCIATION FESTIVAL}

The British Association meetings held in the United Kingdom in September 1999 showed once again that they are capable of surprising the world with announcements of new discoveries and initiatives. At the British Association Festival of Science held at Sheffield, many innovative projects and research activities were launched and highlighted.

One such announcement involved the Telepathy Chip. We were told that British Scientists have been working on such chips that are able to transmit emotions, pain and physical movement impulses between two people. If the research is successful chips will be developed that will allow humans to sense each other's emotions and communicate by thought alone.

At Sheffield, Professor Kevin Warwick of the University of Reading (UK)'s Department of Cybernetics, announced that he had volunteered to become the first human to test the telepathy chip. In some eighteen months he plans to implant in his body the first chip to be wired to a human nervous system.

He told the British Association Festival participants of some of his hopes for such a chip and said that the first signs that the telepathy chip works would come when he writes on a computer screen simply by painting the letters with his forefinger in the space in front of him. This would be accomplished by having a half-inch microprocessor chip implanted in his arm with a sensor collar clamped to a nerve.

$\mathrm{He}$ and other scientists are developing a more sensitive version which has an array of prongs to be clamped to the nerve core. In order to transmit the pain and motor movement stimuli that are picked up from the nerves, both chips contain a transducer to send and receive radio signals.

At the Festival Professor Warwick said that:

"We have conquered the basic technical difficulties of the procedure and are now working on ways to reduce the risk of permanent damage to the nerve if it goes wrong. Our guess is that if two people have the chip one could feel the pain when the other person pricks a finger".

We were told that Professor Warwick's first task would be to send nerve signals to a computer by raising his hand in midair, for example, to switch the light on. Then he will try to reverse the process by receiving signals from the computer that could stimulate his arm into making movements.*

* See also "Cyborgs and Bionics", Robotica 17, part 2, 116-117 (1999).
The potential applications, if the research is successful, are, indeed, wide ranging with its use for improving the quality of life for disabled people an obvious example. One suggestion already considered is that it could be used to provide a way for disabled people to learn from an able bodied person the nerve impulses needed to improve control of limbs.

\section{CHANGE IN THE WORKPLACE}

Many industries involved with automation fail to realise that change is taking place in the workplace. Recently, this has been highlighted by the European Foundation which commissioned a survey in 1996 of 5,800 workplaces in ten European countries. Now a report on the survey based on these survey findings has been made available. A marked difference in the positions of men and women is a key feature of European workplaces, the Foundation tells us, and yet the sexual division of labour is often overlooked in discussions of organisational change. The report shows, however, that innovations in the workplace raise important issues for equal opportunities. While the main focus of the EPOC (Employee Direct Participation in Organisational Change) was on other aspects of direct participation, this report sets out to analyse the gender dimensions of such initiative. It provides a fascinating insight into the gender dimensions of direct participation in organisational change. The analysis includes a wealth of material both from the survey itself and in its findings. There is no doubt that this EPOC report's key findings, which are summarised here, will assist in understanding currant workplace changes. The key findings were:

- Although men and women are equally involved in the practice of direct participation, this takes place in a context of pronounced horizontal and vertical occupational segregation of the sexes. Women are found in particular industries and occupations, often with poorer working conditions than their male counterparts. Lowskilled and repetitious work, for example, is much more likely to be a characteristic of female-dominated establishments. Similarly, workplaces employing women in large proportions are particularly likely to emphasise their reliance on part-time work. They are also less likely to make large capital investments.

- Mixed-sex establishments are the most active practitioners of direct participation in organisational change. These are the workplaces which are most likely to practise multiple and/or high-intensity forms of direct participation; they are therefore implementing more integrated forms of organisational change. 
- There is scope for mixed-sex workplaces to improve equal opportunities, through, for example, the introduction of mixed-sex teamwork in gender-typed areas of production and services; the flattening of male-dominated hierarchies; multiskilling for men and women; and the greater utilisation of the social and communication skills of women.

- Despite this potentially promising scenario, there is no evidence of direct participation being practised with the aim of pressing home equal opportunities objectives in Europe's workplaces. Direct participation has no influence on gender segregation in jobs and working conditions; in some companies, its use even leads to a 'defeminisation' of the labour force.

- Improvements in economic performance due to organisational change are most likely in male dominated companies.

- The provision of training for direct participation indicates that equal opportunities objectives are not being strongly pursued. Training is generally provided along genderstereotyped lines: training in technical skills is mostly offered in male-dominated settings, while 'soft', human skills training is most prevalent in female-dominated environments.

Further information about this report may be obtained from:

European Foundation for the Improvement of Living and Working Conditions,

Wyattville Road, Loughlinstown, Co. Dublin (Ireland).

Tel: + 35312043100

Fax: + 35312826 456/2824209

E-mail: postmaster@eurofound.ie

$\mathrm{EF} / 99 / 35 / \mathrm{EN}$

\section{MARKETING DOMESTIC ROBOTS}

We have been told many times that if only there were viable domestic robots people would clamour to use them in their homes and a new and extensive commercial market would have developed. Of course, such robots exist and some are marketed but they have a long way to go before they reach the levels of the automated washing machine and other such devices that are regarded by householders as essential in the modern home.

A report therefore in the New Scientist about a new robot called Cye may well cause us to think that there must be some progress being made after the many decades when domestic type robots were regarded as figures of fun. The robot is produced by a US company called Probotics which, we are told, is only a small company from Pittsburgh (US). The company tell us that to produce their system they have 'gone back to basics' and have taken some ten years to solve the problems of how to produce a robot that is small and yet mobile and able to perform the many tasks expected of a domestic robotic device. Cye, they say, is an 'automated slave' which is described as looking like a 'dustpan with two cogged wheels'. For communication and control it is designed with a wireless link to a PC. It was apparently necessary, the designers say, to give Cye a 'friendly feel' and to provide it with some sort of warning of its presence.
The result is that it has been equipped with a number of whistles and 'chirps', which have been described by one researcher 'as being like R2-D2 from Star Wars'.

The inventor of Cye, Henry Thorne tells us that "We wanted to make Cye more like a servant where it does its work while staying out of the way."

The developing company say that Cye has no sensors, and is able to 'learn' the positions of obstacles, doorways and walls etc., around the home from software developed for the system and stored in the linked PC. The designers claim that once it has been informed of the limits of its designated area, $C y e$ checks it out and builds a map of it in its computer memory. It does this by counting the number of turns of each of its inbuilt wheels. The wheels themselves are 'patented' and resemble bicycle sprockets and are designed to reduce any slip.

When Cye has stored this information about its domain it can carry out its duties. These it is claimed include such chores as: pulling a trolley loaded with drinks and food; dragging a vacuum cleaner across a room; carrying dishes back to the kitchen. When it has completed its task it quietly returns to its base pod for its batteries to be put on charge.

The company has therefore marketed a domestic robot that it is claimed in its U.S debut 'can serve drinks', 'carry TV dinners', 'escort guests around a home or office', 'vacuum a carpet' and is available for purchase. It will be interesting to learn how well it sells in the market, for the consumer test is the most telling of all in our consumer society.

\section{NEW TECHNOLOGY FOR MEDICINE}

Advances in technology have led to more life saving applications in medicine. A new application described here provides an important aid. Researchers at the Massachusetts Institute of Technology (MIT, USA) have announced a new innovative communications system which will, for example, allow medical doctors to receive and transmit medical information from a patient's bedside at any location. It would mean that in its medical usage the system would allow a General Practioner (GP) to use a mobile phone to take scans and also to view a patient's medical records. The MIT team say that the system, called The Handy 21, will be able to transmit ultra sound and electro-cardiograph signals from the patient's bedside and also receive information about the patient from medical records. It will also work as a cellular telephone, pager, radio, digital television and internet connector. The developers do say, however, that despite this range of functions it will still be no bigger than an ordinary mobile phone because it has a shared processor. The researchers tell us that the next generation of wireless computer networks will be based on the same technology.

The MIT team of researchers is headed by Professor John Guttag who is quoted as believing that:

"Any activity like communications involves processing signals, whether the device is a TV scanner, radio or ultrasound scanner. Historically, the processing of these signals has been done on dedicated, special purpose devices. We decided to take it to the next step and see if we could eliminate the special purpose 
hardware entirely and do all the processing with just off the shelf PC technology. A sensor has only to be added to the Handy 21 to allow it to take different scans, such as magnetic resonance imaging and ultrasound. Users, such as doctors, would plug a sensor into a port on the device and be able to take scans instantly. If a doctor, for example, needed a second opinion, then scans could be sent to another doctor.

It will be like a compressor in the garage that you plug your tools into to inflate your car tyres and do all sorts of different jobs. Nobody has a separate compressor for each job."

The medical application provides the best example of the use of this technology. It would mean that doctors could take all they need to a patient's bedside, wherever that may be, whether in hospital, at home or at any other location, and transmit the data to a base unit at their local hospital for any further analysis. A doctor could then carry out diagnostic tests on patients at any location and send the information to any destination and be able to receive information in return within minutes.

Although this technology has been publicised by the MIT developers in the context of healthcare, the same technology will obviously transfer to numerous other application areas.

\section{ROBOTS WORLDWIDE}

\section{Japan}

In Japan the electronics company Sony have claimed a breakthrough in 'leisure technology'. They are now developing a 'floppy eared poodle' that uses robotics and artificial intelligence to behave as 'cutely' as the designers can achieve. The 'dog' is made of metal and, it is claimed by company spokesman, that it is capable of chasing after a ball and is able to wag its tail when patted by its owner. The ultimate function of the 'creature', we are told, is that its designers have been able to programme it so that it can cock its leg at imaginary lamp posts!

The poodle has been under development for some time, a Tokyo report says, and plans whether to market it are not clear. What is true is that such devices are being produced worldwide and their popularity is extremely high and are not, it would seem, confined to the ownership of young people or, indeed, to children alone.

Despite their designed use, they could still serve as test beds for applications of robotics and certainly artificial intelligence researchers.

\section{United Kingdom}

Games involving robots have become extremely popular worldwide. The UK is no exception and competitions between robots have been extremely attractive not only to the developing robotists but to spectators and to television viewers as well.

Universities, colleges and schools throughout Britain now have robot design teams and many of the products are very skillfully produced. Many are computer designed and do so much to encourage young robotists as well as those who are now established competition figures. UK newspapers as well as the television channels provide coverage. Teams even have their own 'fans'. This is described by the UK's Daily Telegraph (14/9/99) who report that:

"VOLLEYBORG could be the university sport of the $21^{\text {st }}$ century, say some of Britain's brightest robot designers.

The cybernetic challenge is expected to become a mainstay of the robot olympics. Yesterday it saw five university teams of 12-inch-high intelligent robots battling to gain control of and hurl a large, soft ball around table tennis-size volleyball courts. The automatons play in teams of two, operating autonomously to locate the ball and co-operating to throw it into their opposition's court within 40 seconds. Like bats, the robots bounce ultrasound beams around the court to work out their positions and find the ball. Made of Lego bricks and a microprocessor chip at their centres, they play on a two-yard square court."

It was Professor Kevin Warwick and the Cybernetics Department at the University of Reading (UK) who issued the challenge. He is quoted as saying that:

"The challenge is to get the attacker and defender robots to cooperate with one another and to physically locate the soft ball without bumping into the sides of the pitch. It makes them faster ....

I am an ardent Reading football fan and believe me this is much more exciting. Occasionally the robots score, and that is certainly rare for Reading."

\section{United States}

There are now so many 'automated systems' in supermarkets that customers are becoming overwhelmed with new devices and procedures. Even so, at its research centre in New York, USA, IBM have developed a new AI system that has great potential for supermarkets and other food outlets.

The system is called Veggie Vision and it uses artificial intelligence software and a modified photocopier to perform its tasks. The idea of the system's developers is to produce an 'intelligent' device that can, initially at least, be capable of performing some of the tasks that are necessary in shops and supermarkets. The system could soon make the task of recognising and weighing fruit and vegetable produce automatically after they have been selected by a customer. The system is said to be so sensitive that it is able to identify and weigh a particular variety of apple or lettuce. One of the senior officials of IBM who is responsible for retail industries for the corporation says that trials have already been carried out. He says that they have perfected this technology in the laboratory but that the real world is completely different so that it has to be tried out in the targeted retail outlets both in the US and abroad.

The new device Veggie Vision will, it is claimed, replace the current barcode scanners. It is able to take a 3-D scan of an item so that the system is able to build up a profile of it. It can assess its size, shape, texture and even its weight. 
With this observed detail it can compare the data with that stored in a database of the produce, for example, stored in the particular food outlet. This means that a customer's purchase of produce could be matched, in say a supermarket, and the cashier could also see a picture of the item for verification and for pricing. Although the currently developed system is said to be able to differentiate between different types of apple and tomato it has some difficulty in recognising greens. The IBM developers say that:

"We are getting 98\% accuracy in the laboratory, but greens are proving a problem, they all look so similar. Something like lettuce looks much like another lettuce. However, we are getting there, and the system actually learns as it goes along, so over time accuracy will rise."

This is where the implementors of the system need to improvise in the best traditions of AI. If Veggie Vision is uncertain of the vegetable it needs to identify, it will show the cashier its best guesses and ask them to choose the correct one. It is then that the system updates its database to learn how to recognise vegetables that have proved not easy to recognise.

IBM, however, have made the point that the system is experimental and will not be available for at least five years unless there is a breakthrough in technology. At present we are told that the system relies on a large sapphire crystal to form its lens. Not only is it expensive but it is also regarded as too delicate for everyday use. Even so, the developers say that costs are bound to fall when supermarkets worldwide take it on and purchase in bulk.

The philosophy behind this system is that there is a need for such a system because the amount and variety of fruit and vegetables available in our supermarkets has grown enormously. The public seems to demand a modern technological system to deal with the selection and purchase of fresh foods, and IBM are more than ready to supply the supermarket owners with the latest 'intelligent' devices. The question, however, remains, "will customers accept these innovative automated systems?"

\section{TECHNOLOGICAL INNOVATIONS}

\section{Can we make computers from cells?}

A recent report on the Internet (connected@telegraph.co.uk) had the heading 'DNA the logical way ahead'. It was one of many that have appeared worldwide in the last decade concerning the harnessing of cells to form the computers of the future. The general belief is that cells can be made to mimic the functions of computers. In particular, they have been seen as possible replacements for silicon or other material that have been used in our computing machines to produce logical devices. The report gives an insight into the researches of two scientists at the Massachusetts Institute of Technology (USA), Drs Sussman and Knight who want to produce biological cells that are programmable just like processors. To do this, we are told, they need to manipulate what goes on inside to mimic the working of the logic gates in microprocessors. They are encouraged in their work by the fact that cells contain material that has several advantages over silicon. The report says that:

"It has been debugged for far longer than silicon, is used in far more computational devices than silicon and is very easy to reproduce. The material is DNA. DNA coupled with natural selection, is unbeatable when it comes to preserving information. Consider for a moment the cluster of genes called hox. This little marvel tells the cells in an embryo where they are on the body so they know what to grow into, be it legs, lungs, or lymph nodes. Hox appeared first some three billion years ago and has been quietly doing the same job the same way for countless generations."

With this in view, Sussman and Knight of MIT want to obtain DNA to make logic gates. If they could achieve this then obviously, all sorts of biochemical engineering becomes possible.

If DNA can act as logic gates then it will be possible to 'program' cells.

Sussman says that:

"Cells are ultimately a good mechanism for the construction of molecular scale machines that can do other useful tasks. We want to program cells to make these machines for us."

If such advances are possible many other researchers would find this capability most useful. Many research teams, worldwide, are currently looking at the creation of nanoscale devices but lack the means of manufacturing them. Using DNA to produce them may well be the answer. The report does concede, however, that although DNA is a proven media for preserving information it is not naturally logical. What is needed is the ability to manipulate DNA to act in a way that makes it programmable.

Sussman and Knight believe that:

"A cell is essentially a tiny protected chemical reaction chamber. By taking some of the chemical mechanisms within a cell we can make them act more predictably. The main chemical mechanism to control is the protein production line since proteins are the most vital chemical complexes in our bodies. They are used everywhere to make cell walls, carry messages and to catalyse reactions. Genes, the short sections of DNA on the larger helix, tell the rest of the cell how to make amino acids. These are then joined up into chains of up to 100,000 units to make proteins. Once a protein has been built it folds into a characteristic shape and goes to work."

The report from MIT continues to explain how a collection of mechanisms can be used so that a cell can be told what to do.

At this stage the researchers are contemplating using $E$ coli or an even simpler organism called Mycoplasma capricolum. The first logic gate, we are told, that the scientists want to try to mimic is the inverter which turns off into on and vise versa. Combine a couple of these and a NOR gate can be obtained (i.e. If $\mathrm{A}$ and $\mathrm{B}$ are false then $\mathrm{C}$ 
is true). Other gates would then follow.

Obviously, the main challenge is to make one gate and Sussman appears to be confident of this when he says that:

"The actual construction of a gate in itself is not hard-there are many similar naturally occurring mechanisms. What we are working on is being able to make the logic circuits we want reliably."

\section{Automated baggage systems}

Computerised baggage systems were one of the first potential applications for computerisation. Many airports have them together with many other sophisticated systems. Still, however, baggage gets misdirected and lost and many other computerised systems do not always perform as well as they should. Perhaps the innovated system being tested by British Airways (BA) will fare a little better and, finally, solve the problems of baggage that simply goes astray.

A new generation of baggage labels that are fitted with microchips are being tested by BA. The new device has been used on some 22,000 bags that are checked into flights from Manchester and Munich to Heathrow (London) airport over an eight week test period. The trial ended in January 1999 and the results will soon be assessed.

Each baggage tag has an antenna and a pinhead sized silicon chip embedded in the label. A specially designed electronic reader has been produced that is capable of identifying the smart label on each bag as the chip transmits its identifying information to a computer.

The bag is then sent to onward flights or the correct baggage carousel for collection. For the trials the reader is installed at Heathrow Airport. Other airlines are interested in the system and, if it is a success, BA intends to install it throughout its network. Currently, a laser bar code is used to identify and track luggage.

BA says this present method has served the industry well, but now a greater number of bags and distances that have to be moved at large airports demand a new technology.

\section{Autonomous vehicles}

Various self-drive or guided vehicles have been described in these reports, all at different stages of development and potential. There is always an element of doubt about the real future of many projects that have been publicised. Certainly there are few, if any, that are now offered as working everyday systems that can be used by vehicle drivers to supplement or replace their labours.

Now, however, a report in the New Scientist published in 1999 describes a system that can, it is claimed, take control of a car and manoeuvre it out of danger, or relieve tired drivers in rush hour traffic. This it would appear, is a real viable system that is currently being tested by researchers in Germany. An intelligent co-pilot has been fitted to a Volkswagen Passat estate car at the Fraunhofer Institute, Karlsruhe (Germany). In its tests it is reported that the system took charge of the car's accelerator, brakes, steering and automatic gearbox to drive it through busy traffic.

What makes the system so unusual is that when the computer took over it allowed the driver to take his hands off the wheel, his feet off the pedals and his eyes off the road. We are told that the driver could then, if he wished, regain control of the vehicle simply by speaking orders or putting his hands back onto the steering wheel.

The new development is called the Driver Assisted Scheme and its developers are careful not to raise hopes too high about its incorporation into a production car. They believe that it will be some time before the system is working and installed in vehicles produced for sale by the company.

Similar systems called 'co-pilot' systems have been designed to simply regulate the distance between cars on motorways and have no control over steering. This new endeavour is designed, its inventor Frank Heimes says, to take on all driving functions.

He believes that:

"We are heading towards total driver replacement. It will be able to take over the vehicle and avoid a collision where the driver has not noticed a dangerous situation."

The system works by fitting to the vehicle:

- Small rear view mirror mounted video cameras with an $84^{\circ}$ field of view to sense the road and compare the images with the car's on-board computer

- Movement actuators on the steering wheel, gear shift, accelerator and brake pedals to monitor what the car is doing

- Directional sensors on the front wheels and distance sensors on the back wheels.

These devices combine to 'drive' the vehicle with the 'eyes' of the system hidden behind the rearview mirror inside the vehicle. The pair of cameras positioned a similar distance apart to human eyes are there to provide a three-dimensional view of the road ahead, and have a range of $150 \mathrm{ft}$. It is the sensors on the front and rear wheels that determine how far the vehicle has moved and in which direction.

Frank Heimes, the designer states quite clearly that currently the system works on a vehicle travelling at fairly slow speeds and on ideal roads. The report in the New Scientist also raised questions about the legality of using such a system on the roads. It says that the law would have to be changed before automated driving would be allowed. In the United Kingdom, for example, automatic control of steering and brakes of a vehicle is illegal and drivers would be liable for any accidents caused by an autonomous system.

\section{Signature verifying system}

Methods of analysing handwritten signatures have taxed researchers for many years. The ultimate goal of producing an algorithm that is capable of analysing a signature automatically to a high degree of accuracy still beckons. The potential applications are numerous and financially attractive to research groups. It has to some extent become the 'Holy Grail' for computer scientists.

A further contribution to these endeavours is being made by researchers at the Electronic Engineering Laboratory at the University of Kent at Canterbury (UK). Their aim is to 
develop powerful tools to enable handwritten signatures to be automatically analysed. Researchers at the laboratory work on what is called their 'signature verification' project which is focused on applying techniques to increase the reliability with which handwritten signatures can be analysed and automatically authenticated.

The principal investigator of the project, Professor Michael Fairhurst, believes that although this is the main aim it also has a broader long-term potential. He says that:

"An exciting aspect of our work has been the emergence of techniques that are likely to have a very important generic element relevant to a range of problems arising from the processing of handwritten documents, in addition to increasing significantly the diversity of practical solutions where the signature verification can be used effectively."

The researchers at the University of Kent are exploring these new techniques in collaboration with PenOp which is a software company that develops and markets signature processing technology for electronic commerce applications. In order to help refine signature analyses, extensive data collection trials have been undertaken in various public sites including some well known high street stores.

The researchers say that:

"Although the main thrust of our work has been on investigating novel approaches to enhancing the robustness and reliability of automatic signature verification, we have also built on techniques previously established in our group."

The group have explored various avenues to improving signature verification. One of the most effective has been the development of better models to characterise an individual's signature. This has identified a number of techniques for 'validating' a signature model to increase confidence in its power to represent genuine samples.

Another way of optimising decisions about authenticity which has been studied, is the selective exploitation of information that characterises an individual's signing patterns and styles. Perhaps of greatest interest is the approach that is currently attracting most attention. According to Professor Fairhurst this has been one of trying to gain a better understanding of the process whereby forged imitations of a signature are generally attempted. This technique has been explored in public trials, we are told, that have been supported by the collaborating company PenOp.

It is interesting to note how this technique is approached. In a trial, participants are asked to 'imitate' a sample signature under a variety of commonly occurring situations, such as when a genuine signature is available, as with a stolen credit card, or when there is no such signature, for example, when the forger has possession only of a cheque book. Professor Fairhurst says that:

"It is important to understand how different conditions influence the sort of models which might be appropriate for maximising security protection."

The group believe that the future development of efficient techniques for integrating different sources of authenticat- ing evidence is one of the most potentially fruitful areas. They also observe that in addition to exploiting as much signature information as possible from different types of feature extraction processes, improved integration could draw more effectively on different, but complementary biometric measures such as speech and facial characteristics.

Further information about these studies and the programme that has been funded by the Engineering and Physical Science Research Council (EPSRC) can be obtained from the web links:

eleceng.ukc.ac.uk/research/ (University of Kent, UK) www.penop.com (PenOp-software company.)

\section{5. 'Smart' clothing}

A report from researchers at the Georgia Technology Institute's School of Textile and Fibre Engineering describes what is called a 'smart shirt or vest'. This is an example of high tech clothing that has been made from fibre optics and electrically conductive thread. This new material will allow its connection to computer systems in their many and various forms. For example, the scientists say that computer chips can be plugged into the garment allowing its wearer to surf the internet, or for an employer to use a computer system to track a worker's movements.

One important application is the use of smart clothing to monitor the health of the wearer, and to transmit details to a medical centre, hospital or directly to the doctor. In fact, smart clothing was initially developed for military use so that anyone who was wounded or needed attention could be pinpointed on the battlefield and details of injuries etc. could be relayed to the medical services. Other obvious uses are being examined and, in particular, in the civilian sector their use in hospitals, nursing homes and emergency services, including the police and fire brigades, are being actively considered.

Sensors in the clothing can be set to monitor, for example, the wearer's blood flow, body sounds, stress levels and even, the developers' say, create a computer body image. One example given suggests the use of these wearer based systems to monitor a baby for signs of sudden death syndrome. A similar system could be used to carry out checks at a distance on a patient with heart disease.

Currently, we are told, it is the military market which is commanding most attention. The military version that has been produced has a page sized processor that analyses data and transmits it via a satellite. This has been costed at $£ 400,000$ in development charges, but we are assured in its mass produced state it would cost £20-30.

\section{UK RESEARCH PROJECTS}

\section{New optical devices}

A report from the UK's Optoelectronic Systems Integration (OSI) programme gives details of a sponsored project where:

"Practical opportunities for shrinking the size and cutting the cost of optical bar code scanners and read 
heads are being opened up."

We are told that substantial progress towards developing miniature bar code reading systems has been made on the OSI's three year Microengineered Optical Scanners and Read Heads (MORES) project, which was started at the Imperial College's Department of Electrical and Electronic Engineering in 1998. The principal investigator for the MORES' project, Professor Richard Syms, believes that:

"There are formidable problems in designing optical systems to overcome the loss of signal that follows from size reduction, as well as in developing appropriate integrated fabrication processes. However, if our techniques are successful they could create a wide range of chip-based optical reading devices."

Such new microengineering techniques could create new optical reading devices. The report continues by informing us that:

"MORES is initially focusing on 'remote' scanners which operate at a distance from objects, typified by supermarket bar-code scanners. Currently, these cost about $£ 1,000$ each and the smallest read head is a oneinch cube.

By miniaturising and integrating optical and mechanical functions in the read head, scanners could become more mobile and cheaper. This could assist, for example, in providing shoppers with their own scanners.

The MORES approach combines silica-based waveguide optics with moving mechanical components such as scanning mirrors formed by silicon microengineering."

The final system being developed will use a combination of 'pre-lens' and 'post-lens' deflection methods to obtain a 2D multi-line pattern of the object points being scanned.

Pre-lens deflection is being investigated through a prototype consisting of a moving waveguide device, built from a simple etched baseplate and resonant fibre scanner actuated piezo electrically. In this prototype, deflection of the waveguide effectively moves an object point across a lens, so that the image point tracks across the barcode. Professor Syms emphasises that:

"Scan lines of appropriate length have been demonstrated and signals have been recovered from real product barcodes on a wide range of backing materials."

The post-lens avenue is being explored through novel selfassembly techniques that have been developed at Imperial College, London, on an earlier project. The report indicates that:

These construct 3-D micro-optomechanical components by first fabricating flat parts on a wafer consisting of a thin silicon layer separated from the underlying substrate by an oxide film.

After patterning and etching the silicon and removing the underlying oxide, the parts are automatically rotated out-ofplane by the surface tension forces obtained by melting pads of photoresist material. A simple latch mechanism limits the rotation to 45 degrees.
"Mirrors in this system have a base width of one millimetre, which suggests that components of realistic size may be built by this method."

The research is being supported by the UK's Engineering and Physical Sciences Research Council (EPSRC) which is responsible for a successful and wide ranging programme in Information Technology and Computer Science. Readers can obtain more information from the EPSRC:

Contact: e-mail: michelle.kennedy@epsrc.ac.uk. Reseach contact concerning this reported project can be made through:

www.ee.ic.ac.uk/hp/staff/sjwill/optical/OptsemDev.html - Optical and Imperial College Semiconductor Devices Section.

\section{High density chip production}

The research team at the Dundee University's Advanced Materials Centre Scotland, UK., have developed new ways of producing next generation silicon chips. Their project investigated the use of a newly synthesised range of organometallic compounds to make photomasks used in the production of high density chips.

The new processes they have developed are for the manufacture of the high density integrated cicuits which they say, will be required by industry in the future. Professor James Cairns, the project's principal investigator reports that:

"Not only did we demonstrate the effectiveness of this new photomask technology, but during the research we discovered that the compounds could be decomposed directly to metal under ultra-violet (UV) irradiation. This opens up exciting prospects for yielding high-density metal interconnects in a more direct way than previously feasible."

Dundee's new organometallic compounds, it is claimed, can meet the stringent requirements of the mask writing process inherent in the production operations. This is because the new compounds can be deposited from the vapour phase on to fragile X-ray transparent membranes and can be converted to sub-micron metal patterns using a focused electron beam. At the UK's Rutherford-Appleton Laboratory the Xray source has been used to demonstrate that this technology can produce X-ray masks with metal line widths down to less than 60 nanometres. The university say that worldwide patents have been filed to protect the technology and opportunities for exploiting these innovations commercially are being explored.

Further details from the Dundee Microfabrication and Electronic Materials Research Group. - www.dundee.ac. uk/PhysicsEngineering/mcrores.htm.

\section{Mobile computing project}

In the United Kingdom the Engineering and Physical Sciences Research Council (EPSRC) has encouraged research into the support for mobile computing networks. One recently completed project funded by EPSRC has developed a new way of maintaining the quality of complex computer networks. This has been accomplished by Lancas- 
ter University which developed a novel distribution systems architecture which can adapt automatically to meet changing needs of mobile computing environments. In a recent report to the EPSRC the work of Lancaster researchers in producing innovations in their approach was highlighted. Their new distributed systems approach focused on 'reactive services'. These, we are told, can monitor their environment and alter their behaviour on detecting significant changes, such as a drop in the quality of communications links.

Professor Gordon Blair of Lancaster's Distributed Multimedia Research Group (DMRG) is quoted as saying that:

"Future computer environments will include mobile computers with many different levels of connectivity, such as being disconnected or having weak low-speed wireless or fully-connected high speed links."

The reports says that:

A recently completed EPSRC-funded project led by Professor Blair and Dr Nigel Davies demonstrated that the reactive service concept can effectively support mobile computing services by exploiting the differing connectivity levels to maintain specified quality of service (QoS) levels.

A key outcome of the project was the Reactive Adaptive Proxy Placement (RAPP) architecture which operates within the internationally recognised Common Object Request Broker Architecture (CORBA). RAPP supports reactive distributed services using objects that act as proxies on behalf of mobile devices. Proxy objects separate wireless and wired environments, and mediate between different link types.

Professor Blair also notes that:

"Other architectures employing proxies generally do so in a rather static way. RAPP manages proxies dynamically on the fly, e.g. to install an object into a data stream in response to a decrease in QoS.

RAPP, the report says, selects the right proxy to handle a QoS change identified by a monitoring function, say to deal with a video protocol. A 'proxy factory' creates objects of the specified type at the specified location, and a 'proxy trader' locates appropriate factories.

In addition to RAPP, Professor Blair's team has invented a new 'tuple space' paradigm for mobile transactions. It works in this way:

"A tuple is an ordered set of values separated by commas. In this case, they can represent QoS requirements, such as transmission and error rates, battery life and device location. This builds on 'generative communication' ideas originated at Yale University (USA).

The tuple space 'uncouples' sender and receiver in terms of time and space: they do not need to be aware of each other at the same time and do not need to know each others' identities."

Users of such systems will be interested to know that RAPP has been implemented on the OMNIBROKER CORBA platform on SUN/OS and Linux systems. The University researchers have also created a tuple-space platform for Linux, Windows NT and Solaris systems. RAPP and the tuple-space paradigm have been successfully integrated following extensive testing.

The ESRC report* also informs us that this approach builds on the expertise gained at Lancaster University by their study on mobile computing for utilities industries. Further development in collaboration with other groups is envisaged with the assistance of other EPSRC grants such as the Adapt and Open-ORB projects.

\section{WRAPAROUND VR SYSTEMS}

\section{Smartspace system}

United Kingdom researchers from British Telecom (BT) have introduced a new system of 'wraparound screens'. The developers at their research and development at Ipswich (UK), have developed a new system of screens that they believe will form the prototype office of the future. Screens that give almost a $360^{\circ}$ virtual reality could, they believe, replace the office PC and revolutionise home television. They offer great potential in many applications that commercial development companies will soon wish to exploit.

Already the system is to be marketed and made available by the UK company Incorporated Technologies of Tillicoultry, Clackmannanshire. This firm has licenced the design and, we are told, hopes to begin producing a commercial version by early 2000 .

This development is a result of research carried out at BT in its Smartspace Project. It is a system that allows users to experience virtual reality (VR) without the use of what is currently regarded as the essential bulky helmet.

A spokesman for the BT research team says that:

"We looked at the amount of information people have to use and realised that a normal screen is just not right for displaying it all. While a lot of other companies are producing headsets for VR to immerse their users in their systems, we think it is impractical for the office environment. You really need to see and communicate with your colleagues."

The Smartspace system uses two digital projectors behind the user to project a high resolution image on to a huge screen. A smaller touch screen is used to navigate around the system and a fold-out keyboard is also being developed. The whole system is controlled by a standard PC, which is stored behind the user. A video camera on top of the screen allows the user to take part in video conferencing and BT has developed a prototype system allowing doctors to interview patients remotely.

The user, seated in the Smartspace system has the impression of being completely immersed in the PC screen. The entire unit can also swivel around to allow the user to swing the screen and seat around almost 360 degrees the

\footnotetext{
* Report is published in Impact, Issue 23 (1999). Contact with the Lancaster DMRG researchers can be through the Weblink: www.comp.lancs.ac.uk/computing/research/mpg/most/
} 
developers claim.

Other applications of the system, for example, its use by stock brokers, demand this manoeuvrability and function. The developers say:

"Stockbrokers need to access vast amounts of information. By swivelling the chair and screen around, we can give the user the impression of being surrounded by screens, so that a broker can have different market information beside or even behind him."

BT also say that they have discussed the prototype system with military advisors who hope to use it to train soldiers and pilots. The company marketing the system, Incorpo- rated Technologies, say that the initial problem will be the cost. Their spokesman says that:

"To build a full-feature Smartspace system currently costs $£ 42,500$, so we do not expect many companies to replace all their PCs just yet. However, the cost will come down and we are confident that even home users will consider buying it in a few years."

The marketing company have a number of uses in view apart from the designed office version. A home entertainment centre is one consideration and a kiosk-based system is another.

Professor B.H. Rudall

Norbert Wiener Institute and University of Wales (UK.) 